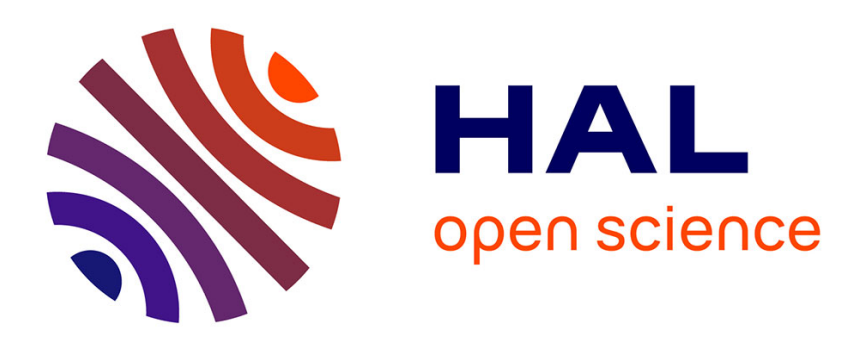

\title{
Data Rate Theorem perspectives on the contribution of extrinsic risk factors to cancer development
}

\author{
Rodrick Wallace
}

\section{To cite this version:}

Rodrick Wallace. Data Rate Theorem perspectives on the contribution of extrinsic risk factors to cancer development. 2016. hal-01276714

\section{HAL Id: hal-01276714 \\ https://hal.science/hal-01276714}

Preprint submitted on 19 Feb 2016

HAL is a multi-disciplinary open access archive for the deposit and dissemination of scientific research documents, whether they are published or not. The documents may come from teaching and research institutions in France or abroad, or from public or private research centers.
L'archive ouverte pluridisciplinaire HAL, est destinée au dépôt et à la diffusion de documents scientifiques de niveau recherche, publiés ou non, émanant des établissements d'enseignement et de recherche français ou étrangers, des laboratoires publics ou privés. 


\title{
Data Rate Theorem perspectives on the contribution of extrinsic risk factors to cancer development
}

\author{
Rodrick Wallace \\ Division of Epidemiology \\ The New York State Psychiatric Institute*
}

February 5, 2016

\begin{abstract}
We explore environmentally-induced tumors in the context of the ongoing conflict between long-evolved cancer control mechanisms and the central flaw of multicellularity, using a number of convergent necessary conditions statistical models based on the Data Rate Theorem linking control and information theories. Multicellular cancer suppression is an inherently unstable dynamic process that can fail through sufficient environmental insult, in particular for humans, involving the synergism of chemical exposures with the chronic inflammation of 'social' exposures that may be seen as accelerated aging.
\end{abstract}

Key Words: chemical exposure; control theory; information theory; social exposure

\section{Introduction}

Recent controversy highlights an ongoing debate on the relative importance of environmental and intrinsic factors in the development and progression of malignancy. Thomasetti and Vogelstein (2015) argue that, in most cases, cancer onset is an inevitable consequence of the natural dynamics of stem cell proliferation, and often a matter of 'bad luck'. Wu et al. (2016) reply that stem cell division per se accounts for minimal rates of cancer in the context of significant environmental insult (Ledford 2015). Here, we use necessary conditions asymptotic theorems from control and information theories to construct a set of statistical models of cancer proliferation focused on the central flaw of multicellularity, which Aktipis et al. (2015) describe as follows:

*Wallace@nyspi.columbia.edu, rodrick.wallace@gmail.com 
Multicellularity requires the suppression of cell-level fitness in order to promote organism level fitness... Cancer represents a breakdown of this multicellular cooperation, with cancer cells 'cheating in ways that can have devastating effects for organism level fitness... Effective multicellularity requires not just cooperation among cells but also mechanisms for suppressing conflict that results from mutations that can enhance cell-level fitness at the expense of the organism... In other words, effective multicellularity requires the suppression of somatic cheating to some degree, and the cancer that results from that cheating.

Nunney (1999) further notes that, as animals become larger, cancer control becomes more difficult at a rate about the $4 / 10$ power of the cell count, consequent, in part, on a synergism with increased lifespan. Large animals must then have tissue-specific cancer control mechanisms whose development may constitute a real barrier to their evolution.

We explore a series of control theory models of the suppression of cheating in multicellular organisms based on the Data Rate Theorem (DRT) that links control and information theories in the specific circumstance of inherent system instability (Nair et al. 2007). Inherently unstable biological systems can respond quickly to environmental or physiological signals and thus may have significant evolutionary advantage under certain powerful selection pressures, as exemplified, in addition to multicellularity itself, by consciousness, blood pressure and gene expression (Wallace 2012).

Control theory has already found a place in the current cancer literature. For example, Schattler and Ledzewicz (2015 Ch 8) explore the Stepanova (1980) model of interaction between tumorigenesis and immune surveillance, commenting that

The competitive interaction between tumor cells and the immune system is complex... and... involves an excessively large number of events with the kinetics of the interplay strongly nonlinear and characterized by multi-stability, i.e., persistence of both benign and malignant scenarios. The possible outcome of this interplay is not only constituted by tumor suppression or tumor outbreak, but there exist many intermediate scenarios.

They go on to examine deviations from nonequilibrium steady states using a linearized model in which stability is defined in terms of a matrix eigenstructure having negative eigenvalues, a standard method whose often complicated symmetries are more fully explored by Golubitsky (1991). Here, via the DRT, we relax the stability conditions and take a more active perspective in which the immune system and other tissue-specific mechanisms (Nunney 1999) exercise ongoing control over the inherent instability of multicellularity in the context of accumulating environmental insult.

Indeed, an emerging strain of opinion on carcinogenesis takes an explicitly systems/network view. As Kreeger and Lauffenburger (2010) put it, 


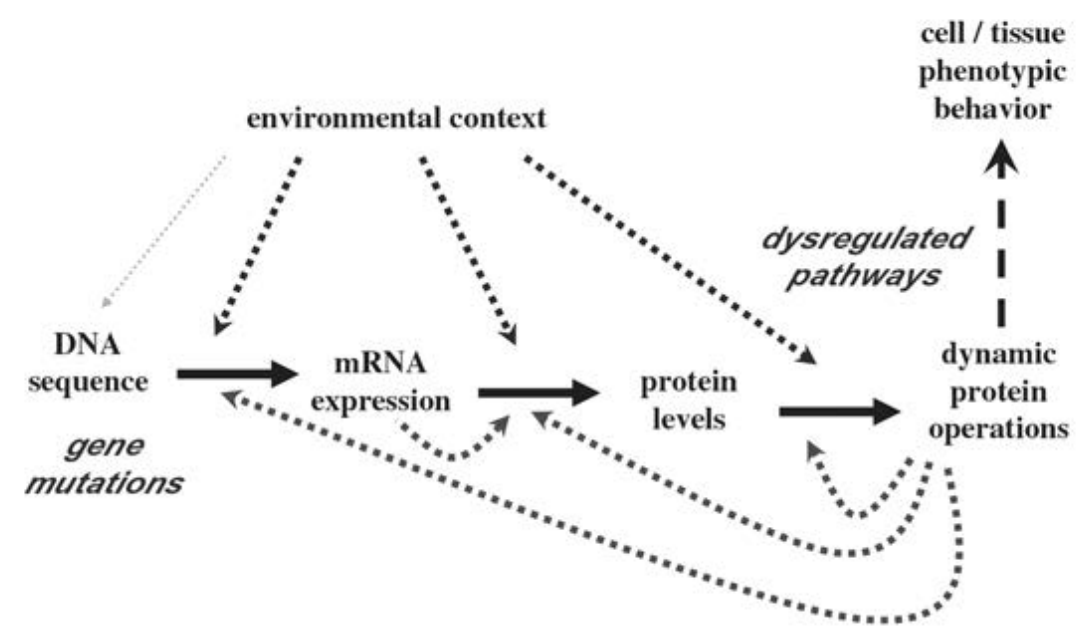

Figure 1: Adapted from Kreeger and Lauffenburger (2010). Environmental context impinges on molecular and genetic regulatory processes to produce malignant phenotypes.

Cancer is now appreciated as not only a highly heterogeneous pathology with respect to cell type and tissue origin but also as a disease involving dysregulation of multiple pathways governing fundamental cell processes such as death, proliferation, differentiation and migration. Thus, the activities of molecular networks that execute metabolic or cytoskeletal processes, or regulate these by signal transduction, are altered in a complex manner by diverse genetic mutations in concert with the environmental context.

Their figure 1, adapted here, schematically illustrates how genetic alterations convolute with environmental context to generate phenotypes, a process in which such context modulates transcriptional, translational and posttranslational processes including epigenetic influences.

A consequence of a systems perspective on cancer biology is the growing understanding that the processes involved are highly dynamic, very subtle, and increasingly defy the standard paradigm of carcinogenesis. As Hlatky and Hahnfeldt (2014) put it,

...[A] vast number of direct observations at the level of tumor progression are showing interactions of cancer cells with themselves and with cells of the host, may not only promote, but also slow, stop, or even reverse a carcinogenesis course... One is confronted with the realization that cancer risk determination from exposures to carcinogenic agents not only requires a multiscale effort to link 
the various levels at which data are available (from molecular to cellular to tissue to epidemiologic), but that the risk itself is influenced at multiple scales, having substantial population- and tissue-level components...

Indeed, as the fundamental study by Folkman et al. (1989) remarks,

A distinguishing feature of most pre-neoplastic lesions is their lack of obvious neovascularization, as compared with the resulting neoplasias which are typically highly angiogenic. The 'switch' from the pre-vascular state to the vascularized stage may be followed by an increase in growth rate and metastasis.

Here, we use the DRT to construct a set of relatively simple statistical models describing how environmental and social exposures drive the expression of malignant phenotypes in complex regulatory networks for which suppression is an ongoing interactive process, with constant low levels of malignancy as a 'normal' condition of multicellularity.

As with regression models, more complicated versions are possible, and these can, perhaps, be fitted to data. And as with all such approaches, in addition to insight, scientific dividends lie in the empirical comparison of similar systems under different, or different systems under similar, conditions and dynamics.

\section{The Data Rate Theorem}

We assume, as do Schattler and Ledzewicz, that the cancer development/cancer control system has been placed in a nonequilibrium - but, in this case, inherently unstable - steady state kept from large-scale explosive tumor growth by a constant flow of externally-imposed control signals. Sufficiently fine-scale search would, however, always find some positive and fluctuating level of malignancy, in this model.

At first order near that steady state, an n-dimensional vector of system parameters at time $t, x_{t}$, determines system development at time $t+1$ according to the relation

$$
x_{t+1}=A x_{t}+\text { Stochastic and Control terms }
$$

where $A$ is an $n \times n$ matrix, and the stochastic and control loop terms are also linear. The essence of the Data Rate Theorem (Nair et al. 2007) in this case is that the rate control information $\mathcal{H}$ needed to prevent explosive instability is determined by the relation

$$
\mathcal{H}>\log \left[\left|\operatorname{det}\left(A^{u}\right)\right|\right] \equiv \alpha_{0}
$$

where det is the determinant and $A^{u}$ is the factored-out submatrix of $A$ having eigenvalues $\geq 1$. Since the left hand side of Eq. (2) is an information measure, the right hand side is interpreted as the rate at which the system generates 
'topological information'. Generalizing to more complex structures, for stability the rate of control information must be greater than the rate at which an inherently unstable system generates topological information.

Given the relatively fixed nature - by evolution and individual development - of the immune/cancer interaction network, the source of 'topological information' is the sum total of 'environmental insult', in a large sense, which we write as ' $\rho$ '. Under such insult, the control system responds, and Eq.(2) can be written as

$$
\mathcal{H}(\rho)>f(\rho) \alpha_{0}
$$

where $f(0) \alpha_{0}$ is to be interpreted as the rate at which the system generates topological information when unperturbed by environmental exposures. What, then, are the functions $\mathcal{H}(\rho)$ and $f(\rho)$ ?

We can approximate $\mathcal{H}(\rho)$ using a Black-Scholes approach (Black and Scholes $1973)$.

\section{The cost of regulation}

Take $\mathcal{H}(\rho)$ as the control information rate 'cost' of stability at the level of environmental insult $\rho$. What is the mathematical form of $\mathcal{H}(\rho)$ under conditions of volatility i.e., variability in $\rho$ proportional to it? Let

$$
d \rho_{t}=g\left(t, \rho_{t}\right) d t+b \rho_{t} d W_{t}
$$

where $d W_{t}$ is taken as white noise and the function $g(t, \rho)$ will 'fall out' of the calculation on the assumption of certain regularities.

Let $\mathcal{H}\left(\rho_{t}, t\right)$ be the minimum needed incoming rate of control information under the Data Rate Theorem, and expand in $\rho$ using the Ito chain rule (Protter 1990)

$$
\begin{aligned}
d \mathcal{H}_{t}=\left[\partial \mathcal{H} / \partial t+g\left(\rho_{t}, t\right) \partial \mathcal{H} / \partial \rho+\right. & \left.\frac{1}{2} b^{2} \rho_{t}^{2} \partial^{2} \mathcal{H} / \partial \rho^{2}\right] d t \\
& +\left[b \rho_{t} \partial \mathcal{H} / \partial \rho\right] d W_{t}
\end{aligned}
$$

Define a quantity $L$ as a Legendre transform of the rate $\mathcal{H}$, by convention having the form

$$
L=-\mathcal{H}+\rho \partial \mathcal{H} / \partial \rho
$$

Since $\mathcal{H}$ is an information index, it is a kind of free energy in the sense of Feynman (2000) and $L$ is a classic entropy measure.

Heuristically, replacing $d X$ with $\Delta X$ in these expressions and applying Eq.(5),

$$
\Delta L=\left(-\partial \mathcal{H} / \partial t-\frac{1}{2} b^{2} \rho^{2} \partial^{2} \mathcal{H} / \partial \rho^{2}\right) \Delta t
$$

As in the classical Black-Scholes model (Black and Scholes 1973), the terms in $g$ and $d W_{t}$ 'cancel out', and the effects of noise are subsumed into the Ito 
correction factor, a regularity assumption making this an exactly solvable but highly approximate model.

The conventional Black-Scholes calculation takes $\Delta L / \Delta T \propto L$. Here, at nonequilibrium steady state, we assume $\Delta L / \Delta t=\partial \mathcal{H} / \partial t=0$, so that

$$
-\frac{1}{2} b^{2} \rho^{2} \partial^{2} \mathcal{H} / \partial \rho^{2}=0
$$

By inspection,

$$
\mathcal{H}=\kappa_{1} \rho+\kappa_{2}
$$

where the $\kappa_{i}$ are nonnegative constants.

Taking the same level of approximation, we assume $f(\rho)$ in Eq.(3) can be similarly expressed as $\kappa_{3} \rho+\kappa_{4}$ so that the stability relation becomes

$$
\frac{\kappa_{1} \rho+\kappa_{2}}{\kappa_{3} \rho+\kappa_{4}}>\alpha_{0}
$$

At low $\rho$ the stability condition is $\kappa_{2} / \kappa_{4}>\alpha_{0}$, and at high $\rho$ it becomes $\kappa_{1} / \kappa_{3}>\alpha_{0}$. If $\kappa_{2} / \kappa_{4} \gg \kappa_{1} / \kappa_{3}$, then at some intermediate value of $\rho$ the essential inequality may be violated, leading to uncontrolled tumor growth. See figure 2 .

A second line of argument leads in a similar direction.

\section{Information bottleneck}

A different possible approach to control system dynamics uses the information bottleneck method of Tishby et al. (1999). The underlying conceit is that the control information needed to stabilize an inherently unstable system, which we have written as $\mathcal{H}$, can be used to define an average distortion measure in a rate distortion calculation. This requires an iterated application of the Rate Distortion Theorem (RDT) (Cover and Thomas 2006) to a control system in which a series of 'control orders' $y^{n}=y_{1}, \ldots, y_{n}$, having probability $p\left(y^{n}\right)$, is sent, and the outcomes monitored as $\hat{y}^{n}=\hat{y}_{1}, \ldots, \hat{y}_{n}$. The distortion measure to be associated with the RDT is now the minimum necessary control information for system stability, written as $\mathcal{H}\left(y^{n}, \hat{y}^{n}\right)$. We can thus, analogously to the standard RDT, define a new average 'distortion' $\hat{\mathcal{H}}$ as

$$
\hat{\mathcal{H}} \equiv \sum_{y^{n}} p\left(y^{n}\right) \mathcal{H}\left(y^{n}, \hat{y}^{n}\right) \geq 0
$$

It then is possible to define a new, iterated, Rate Distortion Function (RDF) $\mathcal{R}(\hat{\mathcal{H}})$ in a standard manner. The central point of any RDF is that it is convex in the distortion measure, and convexity is a very powerful mathematical condition in describing system dynamics (e.g., Ellis 1985).

For simplicity, we take $\mathcal{R}$ to be a Gaussian RDF in $\hat{\mathcal{H}}$,

$$
\begin{array}{r}
R(\hat{\mathcal{H}})=1 / 2 \log \left[\sigma^{2} / \hat{\mathcal{H}}\right] \hat{\mathcal{H}}<\sigma^{2} \\
R(\hat{\mathcal{H}})=0 \hat{\mathcal{H}} \geq \sigma^{2}
\end{array}
$$




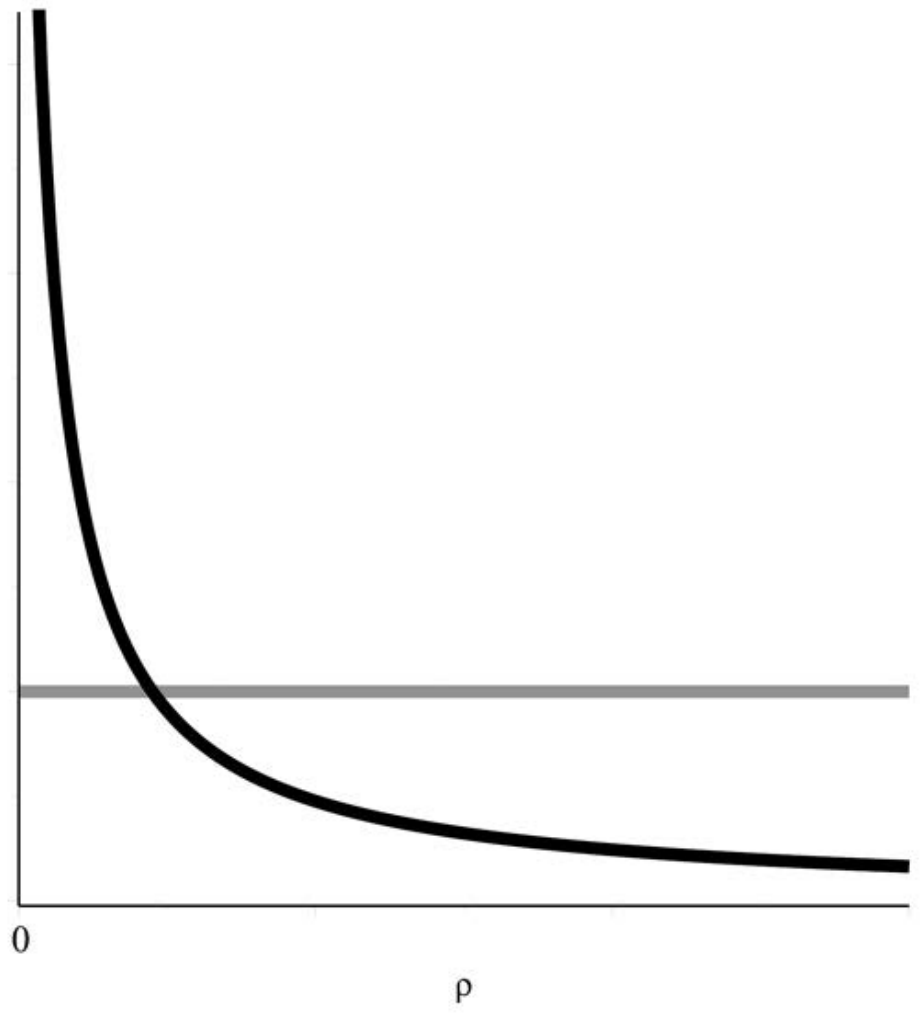

Figure 2: The horizontal line represents the critical limit $\alpha_{0}$. If $\kappa_{2} / \kappa_{4} \gg \kappa_{1} / \kappa_{3}$, at some intermediate value of accumulated environmental insult $\rho$ the relation $\left(\kappa_{1} \rho+\kappa_{2}\right) /\left(\kappa_{3} \rho+\kappa_{4}\right)$ falls below that limit, and malignant growth becomes uncontrolled. 
Again following Feynman (2000), information must be recognized as a form of free energy and a Rate Distortion Function itself, as the minimum channel capacity needed to achieve a given level of average distortion, can be used to define an 'entropy' as the Legendre transform

$$
\mathcal{S}=\mathcal{R}(\hat{\mathcal{H}})-\hat{\mathcal{H}} d \mathcal{R} / d \hat{\mathcal{H}}
$$

Taking Onsager's nonequilibrium thermodynamics perspective (de Groot and Mazur 1984), the dynamics can, in first order, be characterized in terms of the gradients of $\mathcal{S}$, and we invoke an extended analog using the stochastic differential equation (Protter 1990)

$$
\begin{array}{r}
d \hat{\mathcal{H}}_{t}=\left[-\mu d \mathcal{S} / d \hat{\mathcal{H}}_{t}-F(\rho)\right] d t+\beta \hat{\mathcal{H}}_{t} d W_{t} \\
=\left[\frac{\mu}{2 \hat{\mathcal{H}}_{t}}-F(\rho)\right] d t+\beta \hat{\mathcal{H}}_{t} d W_{t}
\end{array}
$$

where $d W_{t}$ is standard white noise, and $F(\rho)$ is a function of accumulated environmental insult $\rho$, that, given an essential 'topological factoring' of the cancer/control network, is the only possible determinant of the rate of generation of system 'topological information'. $\beta$ represents the magnitude of a 'volatility' noise term independent of $\sigma^{2}$ in the definition of $\mathcal{R}$ : higher $\mathcal{H}$, higher stochastic jitter.

Applying the Ito chain rule (Protter 1990) to the expectation of $\hat{\mathcal{H}}_{t}^{2}$, it becomes possible to explore the second moment stability of the system (Khashminskii 2012). A simple calculation finds that the expectation for $\hat{\mathcal{H}}^{2}$ cannot be a real number unless the discriminant of a quadratic equation is nonnegative, giving a necessary condition for stability as

$$
F(\rho) \geq \beta \sqrt{\mu}
$$

We force closure on the model by taking $F(\rho)$ as given by Eq.(10), so that, again,

$$
\frac{\kappa_{1} \rho+\kappa_{2}}{\kappa_{3} \rho+\kappa_{4}} \geq \beta \sqrt{\mu} \equiv \alpha_{0}
$$

with similar restrictions on the constants $\kappa_{i}$ for stability.

Other channel forms will have analogous stability limit conditions on $\rho$ as a consequence of the convexity of the RDF. It is of interest to carry the calculation through for the 'real' channel, having $\mathcal{R}(\hat{\mathcal{H}})=\sigma^{2} / \hat{\mathcal{H}}$.

\section{Turbulence}

A third line of argument, adapted from the air traffic control model of $\mathrm{Hu}$ et al. (2001), involves geodesic streamline flow in a topological quotient space for cellular and tissue dynamics. Given the inherent instability of multicellularity, 'acceptable' dynamic trajectories can be envisioned as constrained to geodesic streamlines in a large 'biospace' $\mathcal{M}$ that have been factored according to a 
maximum microtumor crossection $r$. The equivalence class of such streamlines is written as $W(r)$, and the topological quotient space as $\mathcal{M} / W(r)$, which may be very large indeed. Deviations from geodesics in this quotient space represent biological trajectories having microtumors with crossection greater than $r$. Let $K_{t}$ be an appropriate index of the degree of deviation from such an 'acceptable' geodesic trajectory at time $t$. We can express the dynamics of $K_{t}$, in first order, by the stochastic differential equation

$$
d K_{t} \approx a K_{t} d t+\sigma K_{t} d W_{t}
$$

where $a, \sigma \geq 0$ and $d W_{t}$ represents white noise. Applying the Ito chain rule to $\log \left[K_{t}\right]$, we obtain

$$
d \log \left[K_{t}\right] \approx\left(a-\sigma^{2} / 2\right) d t+\sigma d W_{t}
$$

so that, if $2 a \equiv \alpha_{0}<\sigma^{2}$, the expectation $E[K]_{t} \rightarrow 0$. Our assertion is that the 'noise' $\sigma^{2}$ is again $\mathcal{H}(\rho) / f(\rho)$ from Eq. (3), since $\rho$ is the only possible index for the generation of topological information by biological instability, and $\mathcal{H}$ is the control signal: everything else has been factored out through the construction of geodesics in $\mathcal{M} / W(r)$. We again invoke the closure relation of Eq.(10).

The 'characteristic area' method of Wallace (2016 Section 3) can now be used to extend this model to metastatic tumor proliferation along networks of tissues, as opposed to the dynamics of a biochemical regulatory network studied here.

A slightly more sophisticated model might use the product of the characteristic dimension $r$ and its rate of growth, $d r / d t$, i.e., an index $\hat{r} \propto d r^{2} / d t$, leading to an equivalence class $W(\hat{r})$ and a quotient topology on $\mathcal{M} / W(\hat{r})$.

\section{Cognitive symmetry breaking}

A deeper approach to the dynamics of cancer regulation is via the 'cognitive paradigm' of Atlan and Cohen (1998), who recognized that the immune response is not simply an automatic reflex, but involves active choice of a particular response to insult from a larger repertoire of those possible to it. Such choice reduces uncertainty in a formal manner, and implies the existence of an information source. See Wallace $(2012,2015)$ for details.

Given an information source associated with an inherently unstable, rapidfire cognitive cancer control system - characterized as 'dual' to it - an equivalence class algebra can be constructed by choosing different system origin states $a_{0}$ and defining the equivalence of two subsequent states at times $m, n>0$, written as $a_{m}, a_{n}$, by the existence of high-probability meaningful paths connecting them to the same origin point. Disjoint partition by equivalence class, essentially similar to orbit equivalence classes in dynamical systems, defines a symmetry groupoid associated with the cognitive process. Groupoids represent generalizations of the group concept in which there is not necessarily a product defined for each possible element pair (Weinstein 1996). The simplest example would be a disjoint union of groups. 
The equivalence classes define a set of cognitive dual information sources available to the inherently unstable cancer regulation system, creating a large groupoid, with each orbit corresponding to a transitive groupoid whose disjoint union is the full groupoid. Each subgroupoid is associated with its own dual information source, and larger groupoids will have richer dual information sources than smaller.

Let $X_{G_{i}}$ be the cancer control system's dual information source associated with the groupoid element $G_{i}$, and let $Y$ be the information source associated with incoming environmental stress, in a large sense See Wallace $(2012,2015)$ for details of how environmental regularities imply the existence of an environmental information source.

We can construct a Morse Function (Pettini 2007) as follows.

Let $H\left(X_{G_{i}}, Y\right) \equiv H_{G_{i}}$ be the joint uncertainty of the two information sources. Define a Boltzmann-like pseudoprobability as

$$
P\left[H_{G_{i}}\right]=\frac{\exp \left[-H_{G_{i}} / \kappa \mathcal{H}\right]}{\sum_{j} \exp \left[-H_{G_{j}} / \kappa \mathcal{H}\right]}
$$

where $\kappa$ is an appropriate constant depending on the particular system and its linkages to control signals, and the sum is over the different possible cognitive modes of the full system.

A 'free energy' Morse Function $F$ can be defined as

$$
\exp [-F / \kappa \mathcal{H}] \equiv \sum_{j} \exp \left[-H_{G_{j}} / \kappa \mathcal{H}\right]
$$

Given the inherent groupoid structure, it is possible to apply an extension of Landau's picture of phase transition (Pettini 2007). In Landau's formulation of spontaneous symmetry breaking, phase transitions driven by temperature changes occur as alteration of system symmetry, with higher energies at higher temperatures being more symmetric. The shift between symmetries is highly punctuated in the temperature index, here the minimum necessary control information rate $\mathcal{H}$ under the Data Rate Theorem for unstable control systems. Typically, such arguments involve only a very limited number of possible phases.

Decline in the richness of control information $\mathcal{H}$, or in the ability of that information to influence the system, characterized by $\kappa$, can lead to punctuated decline in the complexity of cognitive process possible within the cancer control system, driving it into a ground state collapse in which tumors proliferate beyond the acceptable topological crossection $r$ (or the expanded index $\left.\hat{r} \propto d r^{2} / d t\right)$.

The essential feature is the integrated environmental insult $\rho$. Most of the topology of the inherently unstable multicellular system has been 'factored out' via the construction of geodesics in a topological quotient space $\mathcal{M} / W(r)$ or $\mathcal{M} / W(\hat{r})$, so that $\rho$ remains the only possible index of the rate of topological information generation for the DRT. Thus, in $\operatorname{Eqs}(19)$ and $(20), \mathcal{H}$ is again replaced by the ratio $\mathcal{H} / f(\rho)$, where $f$ is a dimensionless monotonic increasing positive function. 
For a fixed $\mathcal{H}$, increasing $\rho$ is then equivalent to lowering the 'temperature', and the system passes from high symmetry 'free flow' to different forms of 'crystalline' structure - broken symmetries representing onset of significant tumor growth.

Again, making an exactly-solvable Black-Scholes approximation, the 'cost' of the control information $\mathcal{H}$ can, in first order, be expressed in terms of a linear function of $\rho$ and again, in first order, taking $f(\rho) \approx \kappa_{3} \rho+\kappa_{4}>0$, we obtain an effective 'temperature' as

$$
\kappa \mathcal{H}(\rho) / f(\rho) \approx \frac{\kappa_{1} \rho+\kappa_{2}}{\kappa_{3} \rho+\kappa_{4}}
$$

If $\kappa_{2} / \kappa_{4} \gg \kappa_{1} / \kappa_{3}$, accumulated environmental insult will quickly bring the effective 'temperature' below some critical value, triggering collapse into a dysfunctional ground state of tumor proliferation.

\section{Discussion and conclusions}

Following Eq.(10) et seq., at a low level of cumulative environmental insult $\rho$, the stability condition across a spectrum of mathematical models of tumor proliferation is $\kappa_{2} / \kappa_{4}>$ constant. At a high level, it is asymptotically $\kappa_{1} / \kappa_{3}>$ constant. If $\kappa_{2} / \kappa_{4} \gg \kappa_{1} / \kappa_{3}$, then, according to figure 2 , at some point the cumulative effects of environmental insult may overwhelm the ability of the cancer control system to contain 'normal' conditions of malignancy to an acceptable level, initiating significant tumor growth.

Generalizations of the models might involve better assessment of the functions $\mathcal{H}(\rho)$ and $f(\rho)$ in the various levels of DRT argument. Dynamics will be driven both by normal aging and by the deleterious effect of exposure itself on the control network that can be viewed as accelerated aging, increasing the network constant $\alpha_{0}$ itself. Similar perspectives can, of course, be applied to a variety of pathological developmental trajectories, for example Alzheimer's disease (Wallace 2015).

Something much like this has, of course, long been known. For example, Aktipis and Nesse (2013) write

When considering all cancers, the role of mismatch with modern environments is overwhelming. About one-third of cancers are direct complications of tobacco use, and another third are reported to be results of obesity, inactivity or poor diet... In addition, many cancers are caused by radiation, hormone treatments, environmental exposures, and new pathogens. In contrast, only about $5 \%$ of cancers are products of hereditary genetic abnormalities. Cancer in modern populations is caused mainly not by the innate inadequacies of our bodies, but by exposure to aspects of modern environments for which our bodies are ill prepared. 
Somewhat more generally, however, chemical and, for humans, 'social', exposures, in the context of the mitochondrial burdens of aging, can trigger the chronic inflammation and other mechanisms leading to carcinogenesis (e.g., Wallace 2015 Ch. 8; Wallace and Wallace 2010). An economically sustainable public health attack, as opposed to inordinantly expensive case-by-case disease treatment, involves reduction of such exposures at the population level, most typically through improvements in living and working conditions.

\section{References}

Aktipis, C.A., R. Nesse, 2013, Evolutionary foundations for cancer biology, Evolutionary Applications, 6:144-159.

Aktipis, C.A., A. Boddy, G. Jansen, U. Hibner, M. Hochberg, C. Maley, G. Wilkinson, 2015, Cancer across the tree of life: cooperation and cheating in multicellularity, Philosophical Transactions B, 370:20140219.

Atlan, H., I. Cohen, 1998, Immune information, self-organization, and meaning, International Immunology, 10:711-717.

Black, F., M. Scholes, 1973, The pricing of options and corporate liabilities, Journal of Political Economy, 81:637-654.

Cover, T., J. Thomas, 2006, Elements of Information Theory, Second Edition, Wiley, New York.

de Groot, S., P. Mazur, 1984, Nonequilibrium Thermodynamics, Dover, New York.

Ellis, R., 1985, Entropy, Large Deviations and Statistical Mechanics, Springer, New York.

Feynman, R., 2000, Lectures on Computation, Westview Press, New York.

Folkman, J., K. Watson, D. Ingber, D. Hanahan, 1989, Induction of angiogenesis during the transition from hyperplasia to neoplasia, Nature, 339:58-61.

Golubitsky, M., 1991, Genericity, bifurcation and symmetry. In Aris, R., D. Aronson, H. Swinney (eds.), Patterns and Dynamics in Reactive Media, The IMA Volumes in Mathematics and its Applications, Springer, NY.

Hlatky, L., P. Hahnfeldt, 2014, Beyond the cancer cell: progression-level determinants highlight the multiscale nature of carcinogenic risk, Cancer Research 74:659-664.

Hu, J., M. Prandini, K. Johnasson, S. Sastry, 2001, Hybrid geodesics as optimal solutions to the collision-free motion planning problem. In Di Beneddto M., A. Sangiovanni-Vincentelli (eds.) HSCC 2001, LNCS 2034:305-318.

Khashminskii, R., 2012, Stochastic Stability of Differential Equations, Second Edition, Springer, New York.

Ledford, H., 2015, Cancer studies clash, Nature 528:317.

Kreeger, P., D. Lauffenburger, 2010, Cancer systems biology: a network modeling perspective, Carcinogenesis 31:2-8.

Nair, G., F. Fagnani, S. Zampieri, R. Evans, 2007, Feedback control under data rate constraints: an overview, Proceedings of the IEEE, 95:108-137. 
Nunney, L., 1999, Lineage selection and the evolution of multistage carcinogenesis, Proceedings of the Royal Society B, 266:493-498.

Pettini, M., 2007, Geometry and Topology in Hamiltonian Dynamics, Springer, New York.

Protter,P., 1990, Stochastic Integration and Differential Equations, Springer, New York.

Schattler, H., U. Ledzewicz, 2015, Optimal Control or Mathematical Models of Cancer Therapies: An application of geometric methods, Springer, NY.

Stepanova, N., 1980, Course of the immune reaction during the development of a malignant tumor, Biophysics, 24:917-923.

Tishby, N., F. Pereira, W. Bialek, 1999, The information bottleneck method, 37th Allerton Conference on Communication, Control and Computing, pp. 368377.

Tomasetti, C., B. Vogelstein, 2015, Variation in cancer risk among tissues can be explained by the number of stem cell divisions, Science 347:78-81.

Wallace, R., D. Wallace, 2010, Gene Expression and its Discontents: The social production of chronic disease, Springer, New York.

Wallace, R., 2012, Consciousness, crosstalk, and the mereological fallacy: an evolutionary perspective, Physics of Life Reviews, 9:426-453.

Wallace, R., 2015, An Information Approach to Miochondrial Dysfunction: Extending Swerdlow's hypothesis, World Scientific, Singapore.

Wallace, R., 2016, Subtle noise structures as control signals in high-order biocognition, Physics Letters A, 380:726-729.

Weinstein, A., 1996, Groupoids: unifying internal and external symmetry, Notices of the American Mathematical Association, 43:744-752.

Wu, S., S. Powers, W. Zhu, Y. Hannun, 2016, Substantial contribution of extrinsic risk factors to cancer development, Nature, 529:43-47. 